\title{
Dual-probe 1D hybrid fs/ps rotational CARS for simultaneous single-shot temperature, pressure, and $\mathrm{O}_{2} / \mathrm{N}_{2}$ measurements
}

\author{
David Escofet-Martin ${ }^{1, *}$, Anthony O. Ojo ${ }^{1}$, Joshua Collins ${ }^{1}$, Nils Torge Mecker ${ }^{1}$, Mark \\ LINNE $^{1}$, AND BRIAN PETERSON ${ }^{1}$ \\ ${ }^{1}$ Institute for Multiscale Thermofluids, University of Edinburgh, Edinburgh, UK. \\ *Corresponding author: david.escofet@ed.ac.uk
}

Compiled September 4, 2020

We employ dual-probe one-dimensional (1D) femtosecond (fs)/picosecond (ps) hybrid rotational coherent antiStokes Raman spectroscopy (HRCARS) to investigate simultaneous temperature, pressure, and $\mathrm{O}_{2} / \mathrm{N}_{2}$ measurements for gas-phase diagnostics. The dual-probe HRCARS technique allows for simultaneous measurements from the time and frequency-domain. A novel approach for measuring pressure, which offers high accuracy $(<1 \%)$ and precision $(0.42 \%)$, is presented. The technique is first demonstrated in a chamber for a range of pressures (1-1.5 bar). This technique shows an impressive capability of resolving $1 \mathrm{D}$ pressure gradients arising from a $\mathrm{N}_{2}$ jet impinging on a surface, both in laminar and turbulent conditions. The technique is shown to be capable of resolving single-shot pressure gradients $(0.04 \mathrm{bar} / \mathrm{mm})$ originating from kinetic energy conversion to pressure and resolves characteristic $\mathrm{O}_{2} / \mathrm{N}_{2}$ structures from laminar and turbulent mixing.

(C) 2020 Optical Society of America

http://dx.doi.org/10.1364/ao.XX.XXXXXX

Temperature is a key thermodynamic variable in reactive flows, because chemical reaction rates are exponentially temperature dependent. Spatially resolved pressure relates to molecular motion and forces, and it is critical for aerodynamic studies. Relative species concentrations are essential when evaluating turbulent mixing, which is prevalent in many practical applications where two gases have to be combined. There is thus a need for spatially resolved, one-dimensional (1D) gas-phase measurements of pressure, temperature and species, which would support flow simulations and optimize operation of system components in practical devices such as gas turbines.

Laser techniques for pressure and temperature are nonintrusive [1]. In gas-phase thermometry, coherent anti-Stokes Raman spectroscopy (CARS) is esteemed for measurement pre- cision and accuracy [2]. Historically, nanosecond (ns) CARS provided zero-dimensional (OD) temperature measurements through highly resolved spectra [3], and simultaneous temperature and pressure measurements were performed by utilizing different spectral features in the frequency-domain $[4,5]$.

New technological developments such as high energy picosecond (ps) and femtosecond (fs) lasers have granted the extension of CARS measurements from OD to 1D [6] and to two-dimensional (2D) [7]. These developments permitted timedomain experiments to resolve collisional behavior. Timeresolved CARS has been used to infer Raman coherence decay information $[8,9]$ and temperature $[10,11]$.

Encoding time-domain information into the frequencydomain has been done by chirped-probe-pulse (CPP) CARS [12] but remains complex and limited to OD. Recently, Prince et al [13] introduced hybrid fs/ps CARS (HCARS). This technique employs fs beams for the pump/Stokes and a ps beam for the probe. With fs/ps HCARS, frequency-domain information is time-resolved due to the short ps probe, while time-domain information can be obtained by scanning the probe delay [14, 15]. The significance of this new configuration can be observed in measurements applied in a wide range of pressures, temperatures, and species [14-20]. Multiple-probe HCARS experiments have been demonstrated in $0 \mathrm{D}$ to correct temperature for timevarying collisional environments [21]. The frequency-domain pressure dependence of HCARS has been exploited to measure pressure [22]. Using this pressure dependence and multiple probes, simultaneous pressure and temperature measurements were performed in OD [23] and 1D [24]. However, a trade-off between pressure sensitivity and signal level exists when evaluating pressure [24]. This limits the measurements accuracy and precision. Single-shot temporally and spectrally resolved coherences have been demonstrated in 0D [25], limitations in detector dimensionality complicate 1D measurements.

This Letter introduces a new dual-probe 1D hybrid fs / ps rotational CARS (HRCARS) approach for simultaneous single-shot temperature, pressure, and $\mathrm{O}_{2} / \mathrm{N}_{2}$ ratio measurements. This analysis exploits the strong time-domain pressure dependence of the CARS signal for accurate and precise pressure measurements and overcomes challenges within the frequency-domain.

The dual-probe 1D fs / ps HRCARS technique is demonstrated 
here in two environments. The first is an optically accessible chamber [26] to evaluate the accuracy of the pressure measurements. The second is a high speed $\mathrm{N}_{2}$ jet impinging on a surface, to demonstrate the utility of the technique in capturing 1D pressure gradients.

Figure 1 illustrates the experimental setup for the $\mathrm{N}_{2}$ impinging jet. The setup is modified from previous HRCARS experimental layouts [7] to include two probe beams. The seed (Vitara) of a Ti:sapphire amplifier delivering $35 \mathrm{fs}$ pulses with $7.5 \mathrm{~mJ}$ at 1 $\mathrm{kHz}$ is locked to the ninth harmonic of the oscillator frequency (80 MHz) of a diode pumped Nd:YAG providing 20 ps pulses with $20 \mathrm{~mJ}$ at $50 \mathrm{~Hz}$.

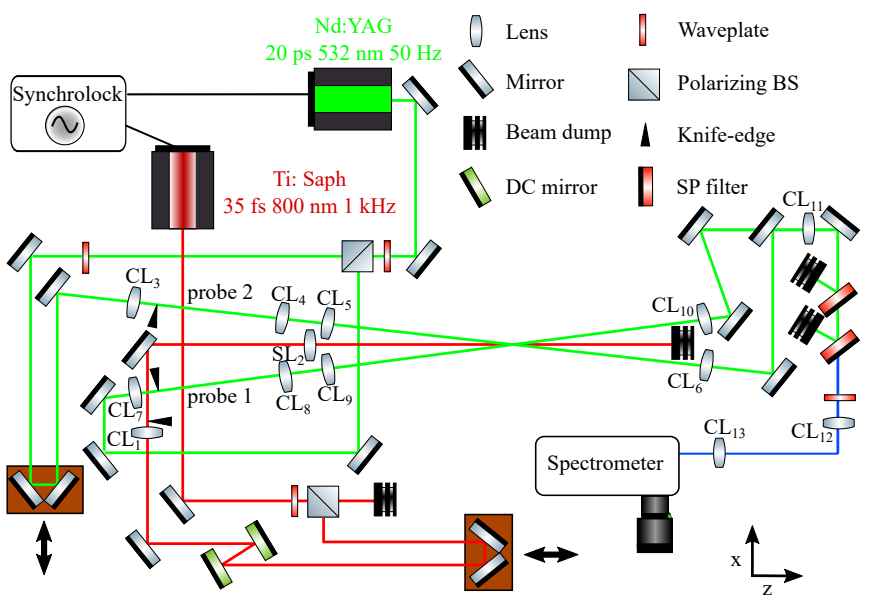

Fig. 1. Schematic of the experimental setup for the $\mathrm{N}_{2}$ impinging jet measurements.

The pump/Stokes energy is adjusted using a variable attenuator [waveplate plus polarizing beam splitter (BS)]. We use dispersion compensation (DC) mirrors to precompensate for dispersion effects caused by the cylindrical lens $(C L)_{1}$ and spherical lens $(\mathrm{SL})_{2}$. A compensation of $-220 \mathrm{fs}^{2}$ provides a near Fourier-transformed pulse after going through the lenses. A combination of a $\mathrm{CL}_{1}\left(\mathrm{f}_{1}=300 \mathrm{~mm}\right)$ and a $\mathrm{SL}_{2}\left(\mathrm{f}_{2}=300 \mathrm{~mm}\right)$ create a laser sheet that intersects the probe beam sheet. The probe beam is split (with a waveplate plus polarizing BS) into 2 probes: $5 \%$ to probe $1\left(\tau_{1} \approx 40 \mathrm{ps}\right)$ and $95 \%$ to probe $2\left(\tau_{2} \approx 270 \mathrm{ps}\right)$. Two translation stages are used to control the relative delays between the three beams. Two pairs of $\mathrm{CLs}_{3,4}\left(\mathrm{f}_{3,4}=150 \mathrm{~mm}\right)$ and $C_{\text {Ls,8 }}\left(f_{7,8}=150 \mathrm{~mm}\right)$, with the optical power aligned with the $y$ axis and separated by $2 f$, are placed in the optical path. The $\mathrm{CLs}_{4,8}$ image a knife-edge into the probe volume with a magnification slightly $<1$. The knife-edge prevents scattering of the beam from the impinging surface. $\mathrm{CLs}_{5,9}\left(\mathrm{f}_{5,9}=250 \mathrm{~mm}\right)$ with the optical power along the $x$ axis create two laser sheets. The laser sheets cross at the probe volume with an angle of $5^{\circ}$ with the pump/Stokes. The beams are then collimated with $\mathrm{CLs}_{6,10}\left(\mathrm{f}_{6,10}=400 \mathrm{~mm}\right)$. The mirrors placed between the probe volume and $\mathrm{CL}_{11}$ are positioned such that both path lengths are the same. The probe volume $\left(0.055 \times 1.2 \times 4.5 \mathrm{~mm}^{3}, \Delta x \mathrm{x}\right.$ $\Delta z \times \Delta y)$ is relay imaged into the entrance of the spectrometer using $\mathrm{CL}_{11}$ and $\mathrm{CL}_{12}\left(\mathrm{f}_{11}=500 \mathrm{~mm}, \mathrm{f}_{12}=250 \mathrm{~mm}\right)$. The anti-Stokes and Stokes signals are separated using two angle-tunable shortpass (SP) filters. The $\mathrm{CL}_{13}\left(\mathrm{f}_{13}=100 \mathrm{~mm}\right)$ focuses the light into the spectrometer. The $0.75 \mathrm{~m}$ spectrometer is equipped with a $2400 \mathrm{gr} / \mathrm{mm}$ grating, which disperses the HRCARS signal onto an electron multiplying charge coupled device (EMCCD). The dispersion is $0.18 \mathrm{~cm}^{-1} /$ pixel with a spectral resolution of 0.75 $\mathrm{cm}^{-1}$ (FWHM). The magnification of the $\mathrm{CLs}_{7,8}$ and $\mathrm{CL}_{11}$ is set to $\approx 0.8$. The EMCCD is binned $2 \times 1$ in the $y$ direction and is operated at $25 \mathrm{~Hz}$ with $1 \mathrm{~ms}$ exposure for a spatial to pixel relationship of $30 \mu \mathrm{m} /$ pixel. Figure 2(a) shows a representative single-shot spectrogram of the CARS intensity, where the two probes are imaged one on top of the other. The resolution of the imaging system is $60 \mu \mathrm{m}$, found by evaluating the sharpness of the imaged knife-edge. A total length of $\approx 4.5 \mathrm{~mm}$ is evaluated from the surface. 1D HRCARS spectrograms are corrected for the finite bandwith of the pump/Stokes pulses by scanning the probe beam delay with respect to the pump/Stokes in an argon environment. A weighted average from these delays is used to correct the experimental spectrograms.
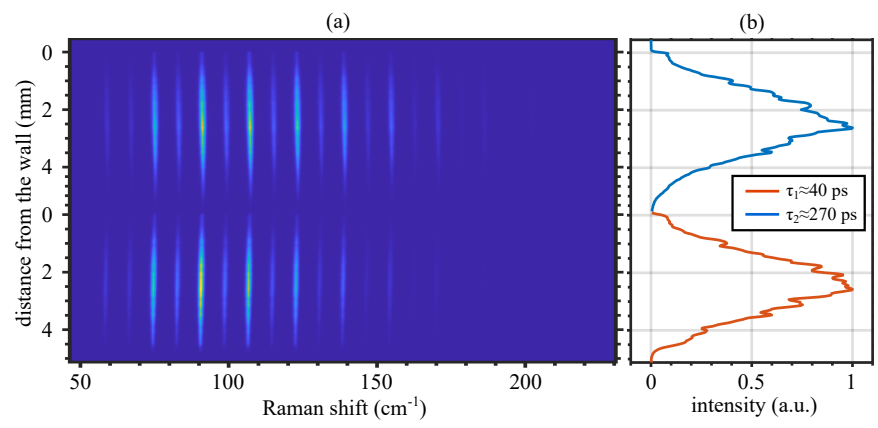

Fig. 2. (a) Single-shot spatially and temporally resolved spectrogram of the dual-probe fs/ps HRCARS near the symmetry axis for the laminar $\mathrm{N}_{2}$ impinging jet (374x1024 pixels). (b) Single-shot spatially and temporally resolved projected intensity of the dual-probe fs/ps HRCARS.

For the chamber experiments, the aforementioned knifeedges (Fig. 1) together with $\mathrm{CLs}_{1,3,4,7,8}$ are removed, and $\mathrm{SL}_{2}$ is substituted for a CL with $\mathrm{f}=300 \mathrm{~mm}$. Due to the increased dispersion from the chamber windows (12 mm fused silica), the number of bounces between the DC mirrors is increased to provide $-440 \mathrm{fs}^{2}$. The measured pulse after passing through the window is $37 \mathrm{fs}$ (FWHM). The energy employed for the pump/Stokes beam is significantly reduced $(<3 \mathrm{~mJ})$ due to supercontinuum light generation in the chamber windows.

The CARS model used here is detailed in $[27,28]$. Pressure dependent linewidths are included with self-broadened linewidths for $\mathrm{N}_{2}$ [20] and $\mathrm{O}_{2}-\mathrm{N}_{2}$ for $\mathrm{O}_{2}$ [29]. For the frequency-domain fitting, a three-dimensional library with varying temperature, pressure, and $\mathrm{O}_{2} / \mathrm{N}_{2}$ ratio was employed.

The spatially resolved intensities for different probe delays shown in Fig. 2(b) are key to studying the time-domain pressure fitting. The intensity from integrating the CARS signal is

$$
I_{C A R S}(T, P, \tau) \propto \int \mathcal{F}\left[P^{(3)}(t, \tau, T, P)\right] d \omega
$$

where $P^{(3)}$ is the third order polarization

$$
P^{(3)}(t, \tau, T, P) \propto\left(\frac{i}{\hbar}\right)^{3} E_{p r}(t-\tau) R_{C A R S}(t, T, P) e^{i \omega_{p r}(t-\tau)} .
$$

$E_{p r}$ is the normalized electric field envelope of the probe, $\tau$ is the relative delay between the probe and the pump/Stokes, and $R_{\text {CARS }}$ is the molecular response function

$$
R_{C A R S}(t, T, P)=\frac{P}{k T} \sum_{J} I_{J ; J+2}(T) \times e^{\frac{t}{\hbar}\left(i \Delta E_{J j J+2}-\frac{1}{2} \Gamma_{J ; J+2}(T, P)\right)},
$$


where $P / k T$ represents the number density effect, $I_{J ; J+2}$ is the Raman transition strength, $\Delta E_{J ; I+2}$ is the frequency of the transitions, and $\Gamma_{J ; J+2}$ is the linewidths.

Given electric fields for pump/Stokes and probe, the $\mathrm{N}_{2}$ HRCARS intensity is a function of probe delay, temperature, and pressure [Eq. (1)-(3)]. Figure 3(a) shows the simulated $\mathrm{I}_{C A R S}$ for two probe delays $\left(\tau_{1}=40 \mathrm{ps}, \tau_{2}=270 \mathrm{ps}\right)$ and the $\mathrm{I}_{\text {ratio }}=\mathrm{I}_{C A R S \tau 1} / \mathrm{I}_{C A R S \tau 2}$ for a range of pressures of 0.9-1.1 bar and temperatures of $280-310 \mathrm{~K}$. The Raman response $\left(R_{C A R S}(t, T, P)\right)$ contains two pressure dependent terms: number density $(P / k T)$ and linewidths $\left(\Gamma_{J ; J+2}(T, P)\right)$. For probe $1\left(\mathrm{I}_{C A R S \tau 1}, \tau_{1}=40 \mathrm{ps}\right)$, the number density contribution dominates the signal intensity for both decreasing temperature and increasing pressure. For probe $2\left(\mathrm{I}_{C A R S \tau 2}, \tau_{2}=270 \mathrm{ps}\right)$, the signal decreases for increasing pressure due to the exponential dependence on linewidth, which overcomes the quadratic signal increase due to number density.
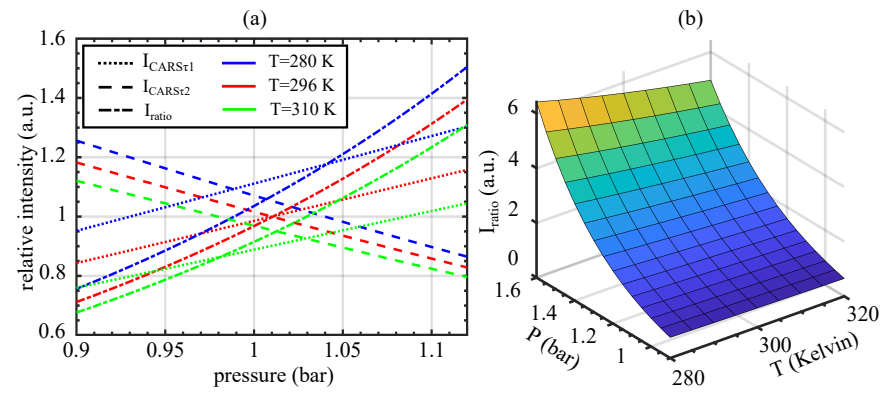

Fig. 3. (a) Simulated intensity as a function of pressure (0.9-1.1 bar) and temperature $(280-310 \mathrm{~K})$ for early probe $\left(\tau_{1}=40 \mathrm{ps}\right)$, late probe $\left(\tau_{2}=270 \mathrm{ps}\right)$ and $I_{\text {ratio }}(\mathrm{b})$ Simulated relation for $\mathrm{I}_{\text {ratio }}$, pressure and temperature.

The $\mathrm{I}_{\text {ratio }}$ for spatially correlated measurements is not a function of number density or pump/Stokes laser energy, and the pressure sensitivity is increased when compared to any of the individual probes. The relation between the simulated $I_{\text {ratio }}$ and the measured $I_{\text {ratio }}$ is not complete until a calibration measurement is performed. This accounts for the energy profiles of both individual probe beams, among other things, and is performed separately for chamber and jet experiments, both at ambient pressure (1.01 bar) and temperature. To better utilize the detector dynamic range, the energy split of probes 1 and 2 is adjusted so that $I_{\text {ratio }} \approx 1$ under the calibration conditions.

Figure $3(\mathrm{~b})$ shows the simulated normalized $\mathrm{I}_{\text {ratio }}$ for a range of temperatures of $280-310 \mathrm{~K}$ and pressures of $0.9-1.6$ bar. The surface plot illustrates the strong sensitivity of the $\mathrm{I}_{\text {ratio }}$ with respect to pressure; a variation from 1 to 1.6 bar produces an $\mathrm{I}_{\text {ratio }}$ change greater than five for this temperature range. The $\mathrm{I}_{\text {ratio }}$ sensitivity with respect to pressure is predominantly a function of $\Delta \tau=\tau_{2}-\tau_{1}$ and can be adjusted to match the expected measured pressure to the dynamic range of the detector.

The fitting procedure starts by assuming ambient pressure in a frequency-domain fit for probe 1 , so we obtain temperature $\left(T_{1}\right)$. This temperature is then used to obtain pressure using the $\mathrm{I}_{\text {ratio }}$-pressure relation. The calculated pressure is then used to refit probe 1 , resulting in the final temperature $(T)$. With the final temperature and the $\mathrm{I}_{\text {ratio }}$-pressure relation, we can obtain the final pressure $(P)$ that has already converged. The statistics displayed in Table 1 are for spatially and time-resolved measurements in the chamber, from evaluating the central 20 spectral rows in 50 shots. The measurements precision for the chamber is hindered compared to the $\mathrm{N}_{2}$ impinging jet experiments due to the reduced pump/Stokes energy $(<3 \mathrm{~mJ})$.

Table 1. Pressure and temperature measurements in the chamber

\begin{tabular}{cccccc}
\hline$\overline{T_{1}}(\mathrm{~K})$ & $\bar{T}(\mathrm{~K})$ & $\bar{P}(\mathrm{bar})$ & $\frac{\sigma_{T}}{\bar{T}}(\%)$ & $\frac{\sigma_{P}}{\bar{P}}(\%)$ & $\overline{P_{\text {trans. }}}($ bar $)$ \\
\hline 296.43 & 296.43 & 1.01 & 1.36 & 0.83 & 1.01 \\
298.59 & 296.59 & 1.22 & 1.14 & 1.29 & 1.21 \\
299.73 & 296.87 & 1.31 & 1.08 & 1.72 & 1.30 \\
300.17 & 296.64 & 1.38 & 1.04 & 1.91 & 1.37 \\
301.30 & 296.83 & 1.48 & 0.94 & 2.44 & 1.49 \\
\hline
\end{tabular}

$T_{1}$ shows a systematic increase of $1.7 \%$ as pressure increases 0.47 bar, while the final calculated temperature $(\bar{T})$ only varies $0.2 \%$ in the studied pressure range. The pressure measurements are benchmarked against a pressure transducer $\left(\overline{P_{\text {trans. }}}\right)$. The

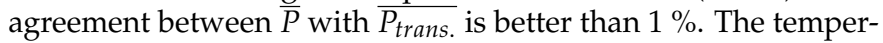
ature precision improves from 1.36 to $0.94 \%$ as pressure increases due to the increased signal from $\mathrm{I}_{C A R S \tau 1}$. The pressure precision decreases from 0.83 to $2.44 \%$ as pressure increases, due to the signal decrease from $\mathrm{I}_{C A R S \tau 2}$.

To demonstrate the utility of the dual-probe HRCARS technique, measurements were applied to a $\mathrm{N}_{2}$ jet impinging on a surface. The surface is curved along the $\mathrm{z}$ direction $(\mathrm{d}=300 \mathrm{~mm})$ for easier optical access. Measurements are performed under two conditions, $\bar{V}=5.2 \mathrm{~m} / \mathrm{s}(\operatorname{Re}=1,420$, laminar $)$ and $\bar{V}=104.2$ $\mathrm{m} / \mathrm{s}(\operatorname{Re}=28,510$, turbulent). The jet inner diameter is $\mathrm{D}=4 \mathrm{~mm}$, and the distance between the jet and the surface is $\mathrm{H}=11 \mathrm{~mm}$, providing a $\mathrm{H} / \mathrm{D}$ ratio of 2.75 . The $4.5 \mathrm{~mm}$ closest to the plate are evaluated for temperature, pressure, and $\mathrm{O}_{2} / \mathrm{N}_{2}$. For every $2 \mathrm{D}$ field, twenty 1D measurements are performed, spaced every 200 $\mu \mathrm{m}$, covering the radial direction $\pm 2 \mathrm{~mm}$ of the jet impingement. The surface is $\left(4 \times 4.5 \mathrm{~mm}^{2}, \Delta x \times \Delta y\right)$, and each averaged field contains the analysis of $(150 \times 20 \times 100) 0.3$ million spectra.

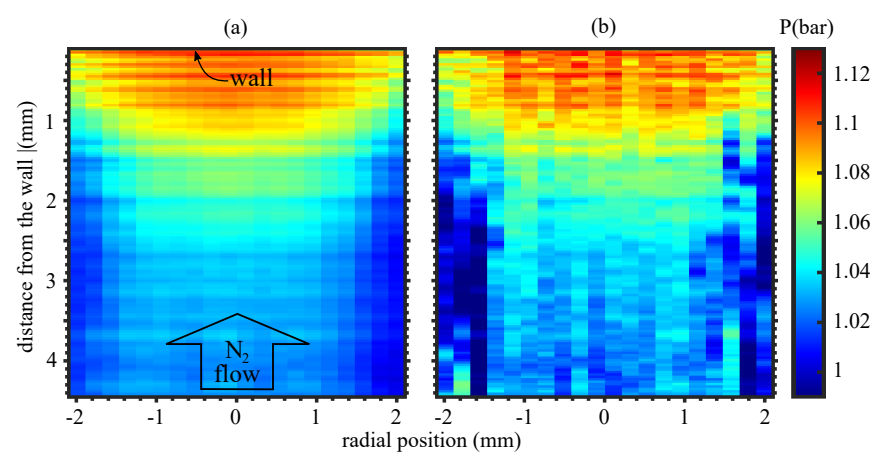

Fig. 4. $2 \mathrm{D}$ pressure field from a turbulent $(\mathrm{Re}=28,510) \mathrm{N}_{2}$ jet impinging on a surface: (a) averaged and (b) single-shot.

The laminar pressure field and the laminar and turbulent temperature fields are uniform, thus not shown here. The pressure and temperature fields obtained from the laminar jet are used to evaluate the measurements precision. The spatio-temporal precision for pressure is $0.42 \%\left(\sigma_{P} / \bar{P}\right)$ and for temperature is $0.62 \%$ $\left(\sigma_{T} / \bar{T}\right)$. Figure 4 shows averaged and instantaneous turbulent pressure fields. The maximum measured pressure is $\approx 1.11$ bar in both the averaged and single-shot fields. This maximum pressure is located near the symmetry axis close to the surface and 
decays both radially and axially. Spatial pressure gradients of the order of $0.04 \mathrm{bar} / \mathrm{mm}$ are well-captured.

To further illustrate the jet behavior, Fig. 5 shows both averaged and single-shot $\mathrm{O}_{2} / \mathrm{N}_{2}$ ratios for both (a), (b) the laminar and (c), (d) the turbulent conditions. The stagnating flow field causes no $\mathrm{O}_{2}$ detection close to the plate. Comparing both $\mathrm{O}_{2} / \mathrm{N}_{2}$ averaged fields, the turbulent field has a $\mathrm{O}_{2} / \mathrm{N}_{2} \approx 0.06$ ratio, a three-fold increase due to enhanced air entrainment near the jet radius. Differences in mixing mechanisms are captured in the single-shot measurements: for the laminar jet, the $\mathrm{O}_{2} / \mathrm{N}_{2}$ measurements show smooth $\mathrm{O}_{2} / \mathrm{N}_{2}$ gradients typical of mixing dominated mostly by diffusion, while the turbulent jet shows fine structure of air pockets from air entrainment, which also demonstrates the exceptional spatial resolution.

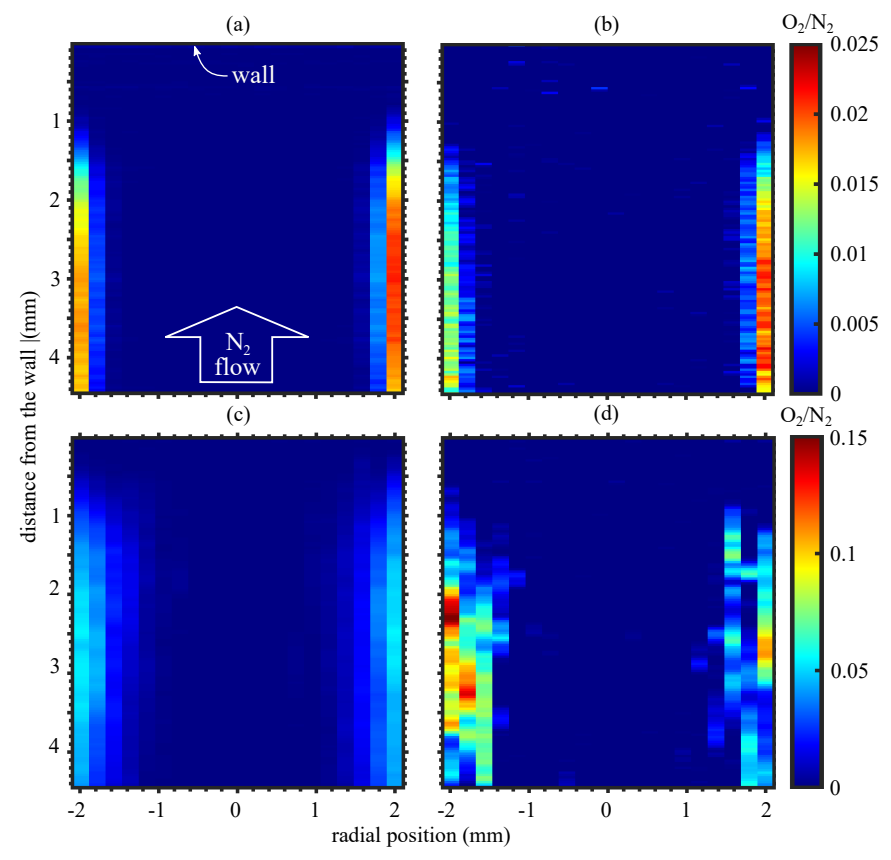

Fig. 5. $2 \mathrm{D} \mathrm{O}_{2} / \mathrm{N}_{2}$ field from a $\mathrm{N}_{2}$ jet impinging on a surface: laminar jet [(a) averaged field, (b) single-shot field] and turbulent jet [(c) averaged field, (d) single-shot field].

In summary, with dual-probe 1D HRCARS, by extracting information from both the time and frequency-domains, we have demonstrated simultaneous pressure, temperature, and $\mathrm{O}_{2} / \mathrm{N}_{2}$ ratio measurements. A new methodology to evaluate pressure leads to high accuracy $(<1 \%)$ for the pressure range 1-1.5 bar, remarkable spatio-temporal temperature precision of $0.62 \%\left(\sigma_{T} / \bar{T}\right)$, unmatched spatio-temporal pressure precision of $0.42 \%\left(\sigma_{P} / \bar{P}\right)$, and fine spatial resolution $(0.055 \times 1.2 \times 0.060$ $\mathrm{mm}^{3}$ ). The pressure measurements are able to describe pressure gradients of the order of $0.04 \mathrm{bar} / \mathrm{mm}$. The $\mathrm{O}_{2} / \mathrm{N}_{2}$ measurements also capture the spatial characteristics from the mixing interface both in laminar and turbulent conditions. Applying this measurement technique in a more challenging environment with higher pressures, temperatures, and species gradients will be the focus of future work. For such studies, anticipated issues such as reduced signal due to fast collisional dephasing at high pressures, unknown collisional partners, and choice of optimal probe delays will be investigated.

Funding. Engineering and Physical Sciences Research Council (EP/P020593/1, EP/P001661/1); European Research Council
(759546).

Disclosures. The authors declare no conflicts of interest.

\section{REFERENCES}

1. A. Y. Chang, B. E. Battles, and R. K. Hanson, Opt. Lett. 15, 706 (1990).

2. S. Roy, J. R. Gord, and A. K. Patnaik, Prog. Energy Combust. Sci. 36, 280 (2010).

3. F. Beyrau, T. Seeger, A. Malarski, and A. Leipertz, J. Raman Spectrosc. 34, 946 (2003).

4. R. E. Foglesong, S. M. Green, R. P. Lucht, and J. C. Dutton, AIAA J. 36, 234 (1998).

5. R. L. Farrow, R. Trebino, and R. E. Palmer, Appl. Opt. 26, 331 (1987).

6. C. J. Kliewer, Y. Gao, T. Seeger, B. D. Patterson, R. L. Farrow, and T. B. Settersten, Appl. Opt. 50, 1770 (2011).

7. A. Bohlin and C. J. Kliewer, J. Chem. Phys. 138 (2013).

8. G. Knopp, P. Beaud, P. Radi, M. Tulej, B. Bougie, D. Cannavo, and T. Gerber, J. Raman Spectrosc. 33, 861 (2002).

9. V. Morozov, S. Mochalov, A. Olenin, V. Tunkin, and A. Kouzov, J. Raman Spectrosc. 34, 983 (2003).

10. S. Roy, P. J. Kinnius, R. P. Lucht, and J. R. Gord, Opt. Commun. 36, 319 (2008).

11. T. Seeger, J. Kiefer, A. Leipertz, B. D. Patterson, C. J. Kliewer, and T. B. Settersten, Opt. Lett. 34, 3755 (2009).

12. D. R. Richardson, R. P. Lucht, W. D. Kulatilaka, S. Roy, and J. R. Gord, Appl. Phys. B: Lasers Opt. 104, 699 (2011).

13. B. D. Prince, A. Chakraborty, B. M. Prince, and H. U. Stauffer, J. Chem. Phys. 125 (2006).

14. A. Bohlin, E. Nordström, B. D. Patterson, P. E. Bengtsson, and C. J. Kliewer, J. Chem. Phys. 137 (2012).

15. J. D. Miller, S. Roy, J. R. Gord, and T. R. Meyer, J. Chem. Phys. 135 (2011).

16. J. D. Miller, S. Roy, M. N. Slipchenko, J. R. Gord, and T. Meyer, Opt. express 19, 15627 (2011).

17. N. T. Mecker, T. L. Courtney, B. D. Patterson, D. Escofet-Martin, B. Peterson, C. J. Kliewer, and M. Linne, J. Opt. Soc. Am. B 37, 1035 (2020).

18. J. D. Miller, C. E. Dedic, and T. R. Meyer, J. Raman Spectrosc. 46, 702 (2015).

19. W. D. Kulatilaka, P. S. Hsu, H. U. Stauffer, J. R. Gord, and S. Roy, Appl. Phys. Lett. 97, 1 (2010).

20. C. J. Kliewer, A. Bohlin, E. Nordström, B. D. Patterson, P. E. Bengtsson, and T. B. Settersten, Appl. Phys. B: Lasers Opt. 108, 419 (2012).

21. B. D. Patterson, Y. Gao, T. Seeger, and C. J. Kliewer, Opt. Lett. 38, 4566 (2013).

22. S. P. Kearney and P. M. Danehy, Opt. Lett. 40, 4082 (2015).

23. C. E. Dedic, A. D. Cutler, and P. M. Danehy, AIAA Scitech 2019 Forum (2019).

24. S. P. Kearney, D. R. Richardson, J. E. Retter, C. E. Dedic, and P. M. Danehy, AIAA Scitech 2020 Forum (2020).

25. A. Hosseinnia, M. Ruchkina, P. Ding, P.-E. Bengtsson, and J. Bood, Opt. Lett. 45, 308 (2020).

26. D. Escofet-Martin, A. O. Ojo, N. T. Mecker, M. A. Linne, and B. Peterson, Proc. Combust. Inst. 38, accepted for publication (2021), doi:10.1016/j.proci.2020.06.097.

27. C. Yang, D. Escofet-Martin, D. Dunn-Rankin, Y. C. Chien, X. Yu, and S. Mukamel, J. Raman Spectrosc. 48, 1881 (2017).

28. C. Yang, P. He, D. Escofet-Martin, J.-B. Peng, R.-W. Fan, X. Yu, and D. Dunn-Rankin, Appl. Opt. 57, 197 (2018).

29. G. Millot, R. Saint-Loup, J. Santos, R. Chaux, H. Berger, and J. Bonamy, The J. Chem. Phys. 96, 961 (1992). 